Supporting Information for Publication

\title{
Removal of thiophene from mixtures with $n$-heptane by selective extraction using deep eutectic solvents
}

\author{
Mohamed K. Hadj-Kali*a ${ }^{a}$, Sarwono Mulyono ${ }^{a}$, Hanee F. Hizaddin ${ }^{b}$, Irfan Wazeer $^{a}$, Lahssen El- blidi ${ }^{a}$, \\ Emad Ali ${ }^{a}$, Mohd. Ali Hashim ${ }^{b}$, Inas M. AlNashef ${ }^{c}$
}

${ }^{a}$ Chemical Engineering Department, College of Engineering King Saud University, P.O.Box 800, Riyadh, 11421, Saudi Arabia.

${ }^{b}$ University of Malaya Center for Ionic Liquids (UMCiL), Department of Chemical Engineering, Faculty of Engineering, University of Malaya, 50603 Kuala Lumpur, Malaysia.

${ }^{c}$ Department of Chemical and Environmental Engineering, Masdar Institute of Science and Technology, Abu Dhabi, United Arab Emirates.

*Corresponding Author. Email address: mhadjkali@ksu.edu.sa

Table S1: List of chemicals used in this work.

\begin{tabular}{lllll}
\hline Name & $\begin{array}{l}\text { Chemical } \\
\text { Formula }\end{array}$ & $\begin{array}{l}\text { Purity } \\
(\text { wt \%) }\end{array}$ & Supplier & Country \\
\hline Thiophene & $\mathrm{C}_{4} \mathrm{H}_{4} \mathrm{~S}$ & 99.5 & Acros Organics & Belgium \\
$n$-heptane & $\mathrm{C}_{7} \mathrm{H}_{16}$ & 99.0 & Scharlau & Spain \\
Ethylene glycol & $\mathrm{C}_{2} \mathrm{H}_{6} \mathrm{O}_{2}$ & 99.0 & Avonchem & England \\
Triethylene glycol & $\mathrm{C}_{6} \mathrm{H}_{14} \mathrm{O}_{4}$ & 99.0 & Sigma Aldrich & USA \\
Sulfolane & $\mathrm{C}_{4} \mathrm{H}_{8} \mathrm{O}_{2} \mathrm{~S}$ & 99.0 & Acros Organics & Belgium \\
Tetrabutylammonium bromide & $\mathrm{C}_{16} \mathrm{H}_{36} \mathrm{BrN}$ & 98.0 & LobaChemie & India \\
Methyltriphenylphosphonium bromide & $\mathrm{C}_{19} \mathrm{H}_{18} \mathrm{BrP}$ & 98.0 & Acros Organics & Belgium \\
\hline
\end{tabular}

Table S2: List of DESs used in this work.

\begin{tabular}{llll}
\hline \multicolumn{1}{c}{ Salt } & \multicolumn{1}{c}{ HBD } & Ratio & \multicolumn{1}{c}{ Abbreviation } \\
\hline Tetrabutylammonium bromide & Ethylene glycol & $1: 4$ & TBAB/EG (1:4) \\
Methyltriphenylphosphonium bromide & Ethylene glycol & $1: 4$ & MeTPPBr/EG (1:4) \\
Tetrabutylammonium bromide & Triethylene glycol & $1: 4$ & TBAB/TrEG (1:4) \\
Tetrabutylammonium bromide & Sulfolane & $1: 7$ & TBAB/Sul (1:7) \\
\hline
\end{tabular}


Table S3: Composition of tie-lines (molar fraction) and the distribution ratio and selectivity data for the ternary system at $25^{\circ} \mathrm{C}$ and atmospheric pressure.

\begin{tabular}{|c|c|c|c|c|c|c|c|}
\hline \multicolumn{3}{|c|}{ Top Layer } & \multicolumn{3}{|c|}{ Bottom Layer } & \multirow[b]{2}{*}{$D$} & \multirow[b]{2}{*}{$S$} \\
\hline $\mathrm{x}_{1}$ & $\mathrm{x}_{2}$ & $\mathrm{x}_{3}$ & $\mathrm{x}_{1}$ & $\mathrm{x}_{2}$ & $\mathrm{x}_{3}$ & & \\
\hline \multicolumn{8}{|c|}{ thiophene (1) $+n$-heptane (2) + TBAB/EG (1:4) (3) } \\
\hline 0.092 & 0.908 & 0.000 & 0.030 & 0.010 & 0.960 & 0.325 & 30.218 \\
\hline 0.197 & 0.803 & 0.000 & 0.046 & 0.011 & 0.943 & 0.233 & 17.776 \\
\hline 0.287 & 0.712 & 0.000 & 0.069 & 0.013 & 0.918 & 0.241 & 13.510 \\
\hline 0.378 & 0.622 & 0.000 & 0.089 & 0.011 & 0.900 & 0.235 & 13.065 \\
\hline 0.4600 & 0.540 & 0.000 & 0.109 & 0.009 & 0.881 & 0.238 & 13.590 \\
\hline 0.552 & 0.448 & 0.000 & 0.146 & 0.010 & 0.844 & 0.265 & 11.567 \\
\hline 0.647 & 0.353 & 0.000 & 0.174 & 0.008 & 0.817 & 0.269 & 11.185 \\
\hline 0.737 & 0.263 & 0.000 & 0.213 & 0.009 & 0.778 & 0.289 & 8.789 \\
\hline \multicolumn{8}{|c|}{ thiophene $(1)+n$-heptane $(2)+\mathrm{MeTPPBr} / \mathrm{EG}(1: 4)(3)$} \\
\hline 0.105 & 0.895 & 0.000 & 0.039 & 0.017 & 0.944 & 0.373 & 20.195 \\
\hline 0.199 & 0.801 & 0.000 & 0.071 & 0.008 & 0.921 & 0.356 & 37.537 \\
\hline 0.288 & 0.712 & 0.000 & 0.084 & 0.008 & 0.908 & 0.290 & 24.807 \\
\hline 0.393 & 0.607 & 0.000 & 0.106 & 0.010 & 0.884 & 0.271 & 16.536 \\
\hline 0.490 & 0.510 & 0.000 & 0.138 & 0.007 & 0.855 & 0.282 & 19.908 \\
\hline 0.583 & 0.417 & 0.000 & 0.157 & 0.005 & 0.838 & 0.270 & 21.229 \\
\hline 0.686 & 0.314 & 0.000 & 0.172 & 0.004 & 0.824 & 0.251 & 17.593 \\
\hline 0.797 & 0.203 & 0.000 & 0.202 & 0.005 & 0.793 & 0.254 & 10.117 \\
\hline \multicolumn{8}{|c|}{ thiophene $(1)+n$-heptane (2) + TBAB/TrEG (1:4) (3) } \\
\hline 0.108 & 0.892 & 0.000 & 0.050 & 0.008 & 0.942 & 0.464 & 51.945 \\
\hline 0.190 & 0.810 & 0.000 & 0.077 & 0.009 & 0.914 & 0.406 & 37.513 \\
\hline 0.323 & 0.677 & 0.000 & 0.107 & 0.020 & 0.873 & 0.332 & 11.160 \\
\hline 0.411 & 0.589 & 0.000 & 0.133 & 0.015 & 0.852 & 0.323 & 12.298 \\
\hline 0.525 & 0.475 & 0.000 & 0.163 & 0.013 & 0.825 & 0.310 & 11.771 \\
\hline 0.613 & 0.387 & 0.000 & 0.188 & 0.016 & 0.796 & 0.307 & 7.3330 \\
\hline 0.698 & 0.302 & 0.000 & 0.211 & 0.009 & 0.780 & 0.302 & 10.702 \\
\hline \multicolumn{8}{|c|}{ thiophene (1) $+n$-heptane (2) + TBAB/Sul (1:7) (3) } \\
\hline 0.092 & 0.908 & 0.000 & 0.069 & 0.016 & 0.915 & 0.753 & 41.869 \\
\hline 0.174 & 0.826 & 0.000 & 0.115 & 0.019 & 0.866 & 0.659 & 28.947 \\
\hline 0.232 & 0.768 & 0.000 & 0.178 & 0.016 & 0.807 & 0.764 & 37.632 \\
\hline 0.322 & 0.678 & 0.000 & 0.226 & 0.019 & 0.755 & 0.703 & 24.863 \\
\hline 0.403 & 0.597 & 0.000 & 0.276 & 0.029 & 0.695 & 0.683 & 13.834 \\
\hline 0.472 & 0.528 & 0.000 & 0.333 & 0.021 & 0.646 & 0.705 & 17.564 \\
\hline 0.562 & 0.438 & 0.000 & 0.370 & 0.021 & 0.609 & 0.659 & 13.770 \\
\hline
\end{tabular}


Table S4. The data of distribution ratio and selectivity

\begin{tabular}{|c|c|c|c|c|c|}
\hline \# & Solvent & $\mathrm{T}(\mathrm{K}) ; \mathrm{P}(\mathrm{kPa})$ & $\mathrm{D}$ & $S$ & Ref \\
\hline 1 & [BMPYR][FAP] & $298.15 ; 101.25$ & 5.46 & 57.9 & [S1] \\
\hline 2 & [BMPYR][TCB] & $298.15 ; 101.25$ & 3.31 & 74.9 & {$[\mathrm{~S} 1]$} \\
\hline 3 & [BMPYR][TCM] & $298.15 ; 101.25$ & 3.47 & 133.4 & {$[\mathrm{~S} 1]$} \\
\hline 4 & [DMIM][MP] & $298.15 ; 101.25$ & 0.42 & 1756.2 & {$[\mathrm{~S} 2]$} \\
\hline 5 & [EMIM][SCN] & $298.15 ; 101.25$ & 0.64 & 1598.5 & {$[\mathrm{~S} 2]$} \\
\hline 6 & [EMIM][SCN] & $305.15 ; 101.25$ & 0.69 & 497.3 & {$[\mathrm{~S} 2]$} \\
\hline 7 & [TEMA][MeSO4] & $298.15 ; 101.25$ & 0.08 & 102.0 & {$[\mathrm{~S} 2]$} \\
\hline 8 & {$[$ Emim $][\mathrm{FAP}]$} & $298.15 ; 101.25$ & 4.00 & 95.1 & {$[\mathrm{~S} 3]$} \\
\hline 9 & {$\left[\mathrm{C}_{2} \mathrm{OHmim}\right][\mathrm{FAP}]$} & $298.15 ; 101.25$ & 2.20 & 168.0 & {$[\mathrm{~S} 3]$} \\
\hline 10 & {$\left[\mathrm{COC}_{2} \mathrm{~N}_{1,1,2}\right][\mathrm{FAP}]$} & $298.15 ; 101.25$ & 4.00 & 90.8 & {$[\mathrm{~S} 3]$} \\
\hline 11 & {$\left[\mathrm{COC}_{2} \mathrm{mMOR}\right][\mathrm{FAP}]$} & $298.15 ; 101.25$ & 2.70 & 109.0 & [S4] \\
\hline 12 & {$\left[\mathrm{COC}_{2} \mathrm{mPIP}\right][\mathrm{FAP}]$} & $298.15 ; 101.25$ & 4.00 & 56.8 & [S4] \\
\hline 13 & {$\left[\mathrm{COC}_{2} \mathrm{mPYR}\right][\mathrm{FAP}]$} & $298.15 ; 101.25$ & 3.10 & 56.6 & [S4] \\
\hline 14 & {$\left[\mathrm{COC}_{2} \mathrm{mMOR}\right]\left[\mathrm{NTf}_{2}\right]$} & $298.15 ; 101.25$ & 1.76 & 104.3 & {$[\mathrm{~S} 5]$} \\
\hline 15 & {$\left[\mathrm{COC}_{2} \mathrm{mPIP}\right]\left[\mathrm{NTf}_{2}\right]$} & $298.15 ; 101.25$ & 2.64 & 62.9 & {$[\mathrm{~S} 5]$} \\
\hline 16 & {$\left[\mathrm{COC}_{2} \mathrm{mPYR}\right]\left[\mathrm{NTf}_{2}\right]$} & $298.15 ; 101.25$ & 2.39 & 70.6 & {$[\mathrm{~S} 5]$} \\
\hline 17 & [EMIM][TCM] & $298.15 ; 101.25$ & 2.38 & 233.7 & [S6] \\
\hline 18 & [EMIM][TCM] & $308.15 ; 101.25$ & 1.94 & 164.7 & [S6] \\
\hline 19 & {$\left[\mathrm{C}_{2} \mathrm{mim}\right]\left[\mathrm{EtSO}_{4}\right]$} & $298.15 ; 101.25$ & 1.03 & 77.8 & [S7] \\
\hline 20 & {$\left[\mathrm{C}_{8} \mathrm{mim}\right]\left[\mathrm{BF}_{4}\right]$} & $298.15 ; 101.25$ & 1.61 & 10.5 & {$[\mathrm{~S} 8]$} \\
\hline 21 & {$[\mathrm{BMPYR}]\left[\mathrm{CF}_{3} \mathrm{SO}_{3}\right]$} & $308.15 ; 101.25$ & 1.80 & 72.0 & [S9] \\
\hline 22 & [HMIM][TCB] & $308.15 ; 101.25$ & 2.68 & 42.5 & [S9] \\
\hline 23 & Sulfolane & $313.15 ; 101.25$ & 0.77 & 46.0 & [S10] \\
\hline 24 & TBAB/EG (1:4) & $298.15 ; 101.25$ & 0.33 & 30.2 & $* *$ \\
\hline 25 & MeTPPBr/EG (1:4) & $298.15 ; 101.25$ & 0.36 & 37.9 & $* *$ \\
\hline 26 & TBAB/TrEG $(1: 4)$ & $298.15 ; 101.25$ & 0.46 & 51.9 & $*$ \\
\hline 27 & TBAB/Sul (1:7) & $298.15 ; 101.25$ & 0.75 & 41.9 & $* *$ \\
\hline
\end{tabular}

Table S5. Normal boiling temperature of thiophene and different HBDs used in this work

\begin{tabular}{clcc}
\hline$\#$ & Chemicals & Boiling Point $\left({ }^{\circ} \mathrm{C}\right)$ & Ref. \\
\hline 1 & Thiophene & 84.0 & {$[\mathrm{~S} 11]$} \\
2 & Ethylene glycol & 197.2 & {$[\mathrm{~S} 12]$} \\
3 & Triethylene glycol & 285.0 & {$[\mathrm{~S} 13]$} \\
4 & Sulfolane & 287.3 & {$[\mathrm{~S} 14]$} \\
\hline
\end{tabular}


Abbreviations:

[BMPYR][FAP]

[BMPYR][TCB]

1-butyl-1-methylpyrrolidinium tris(pentafluoroethyl) trifluorophosphate

[BMPYR][TCM]

[DMIM][MP]

[EMIM][SCN]

[TEMA][MeSO4]

[emim][FAP]

[C2OHmim][FAP]

1-butyl-1-methylpyrrolidinium tetracyanoborate

1-butyl-1-methylpyrrolidinium tricyanomethanide

1,3-Dimethylimidazolium methylphosphonate

1-ethyl-3-methylimidazolium thiocyanate

tris-(2-hydroxyethyl)-methylammoniummethylsulfate

trisfluorotris(perfluoroethyl)phosphate; 1-ethyl-3-methylimidazol-3-ium

$(1-$

Ethyl-3-methylimidazolium trisfluorotris(perfluoroethyl)phosphate)

trifluorotris(perfluoroethyl)phosphate; 2-(3-methylimidazol-3-ium-1-yl)ethanol

(1-(2-Hydroxyethyl)-3-methylimidazolium

trifluorotris(perfluoroethyl)phosphate)

[COC2N1,1,2][FAP] trifluorotris(perfluoroethyl)phosphate; ethyl-dimethyl-(2-methoxyethyl)azanium (Ethyl-dimethyl-(2-methoxyethyl)ammonium

trifluorotris(perfluoroethyl)phosphate)

[COC2mMOR][FAP] trifluorotris(perfluoroethyl)phosphate; 4-(2-methoxyethyl)-4-methylmorpholin-4ium(4-(2-Methoxyethyl)-4-

methylmorpholiniumtrifluorotris(perfluoroethyl)phosphate)

[COC2mPIP][FAP] trifluorotris(perfluoroethyl)phosphate; 1-(2-methoxyethyl)-1-methylpiperidin-1ium(1-(2-Methoxyethyl)-1-

methylpiperidiniumtrifluorotris(perfluoroethyl)phosphate)

[COC2mPYR][FAP] trifluorotris(perfluoroethyl)phosphate; 1-(2-methoxyethyl)-1-methylpyrrolidin-1-

ium(1-(2-methoxyethyl)-1-

methylpyrrolidiniumtrifluorotris(perfluoroethyl)phosphate)

[COC2mMOR][NTf2] (4-(2-methoxyethyl)-4-methyl-morpholiniumbis(trifluoromethylsulfonyl)-amide

[COC2mPIP][NTf2] 1-(2-methoxyethyl)-1-methylpiperidiniumbis-(trifluoromethylsulfonyl)-amide

[COC2mPYR][NTf2] 1-(2-methoxyethyl)-1-methylpyrrolidiniumbis(trifluoromethylsul-fonyl)-amide

[EMIM][TCM] 1-ethyl-3-methylimidazolium tricyanomethanide

[C2mim][EtSO4] 1-ethyl-3-methylimidazolium ethylsulfate

[C8mim][BF4]

[BMPYR][CF3SO3]

[HMIM][TCB]

1-methyl-3-octylimi-dazolium tetrafluoroborate

butyl-1-methylpyrrolidinium tricyanomethanide

1-hexyl-3-methylimidazolium tetracyanoborate 


\section{References:}

[S1] Domańska, U.; Lukoshko, E. V.; Królikowski, M., Separation of thiophene from heptane with ionic liquids. The Journal of Chemical Thermodynamics 2013, 61, 126-131.

[S2] Kędra-Królik, K.; Fabrice, M.; Jaubert, J.-N., Extraction of thiophene or pyridine from n-heptane using ionic liquids. Gasoline and diesel desulfurization.Industrial \& Engineering Chemistry Research 2011, 50, (4), 2296-2306.

[S3] Marciniak, A.; Wlazło, M., Ternary (liquid + liquid) equilibria of \{trifluorotris(perfluoroethyl)phosphate based ionic liquids + thiophene + heptane : Part 2. The Journal of Chemical Thermodynamics 2015, 86, 196-201.

[S4] Marciniak, A.; Królikowski, M., Ternary (liquid+ liquid) equilibria of \{trifluorotris (perfluoroethyl) phosphate based ionic liquids+ thiophene+ heptane\}. The Journal of Chemical Thermodynamics 2012, $49,154-158$.

[S5] Marciniak, A.; Królikowski, M., Ternary liquid-liquid equilibria of bis(trifluoromethylsulfonyl)amide based ionic liquids + thiophene $+n$-heptane. The influence of cation structure.Fluid Phase Equilibria2012, 321, 59-63.

[S6] Królikowski, M.; Walczak, K.; Domańska, U., Solvent extraction of aromatic sulfur compounds from n-heptane using the 1-ethyl-3-methylimidazolium tricyanomethanide ionic liquid. The Journal of Chemical Thermodynamics 2013, 65, 168-173.

[S7] Alonso, L.; Arce, A.; Francisco, M.; Soto, A., Thiophene separation from aliphatic hydrocarbons using the 1-ethyl-3-methylimidazolium ethylsulfate ionic liquid. Fluid Phase Equilibria2008, 270, (1), 97-102.

[S8] Alonso, L.; Arce, A.; Francisco, M.; Soto, A., Solvent extraction of thiophene from n-alkanes $\left(\mathrm{C}_{7}\right.$, $\mathrm{C}_{12}$, and $\left.\mathrm{C}_{16}\right)$ using the ionic liquid $\left[\mathrm{C}_{8} \mathrm{mim}\right]\left[\mathrm{BF}_{4}\right]$. The Journal of Chemical Thermodynamics 2008, 40, (6), 966-972.

[S9] Domańska, U.; Walczak, K.; Królikowski, M., Extraction desulfurization process of fuels with ionic liquids. The Journal of Chemical Thermodynamics 2014, 77, 40-45.

[S10] Hansmeier, A. R.; Meindersma, G. W.; de Haan, A. B., Desulfurization and denitrogenation of gasoline and diesel fuels by means of ionic liquids. Green Chemistry 2011, 13, (7), 1907-1913.

[S11] Mishra, R.; Jha, K.; Kumar, S.; Tomer, I., Synthesis, properties and biological activity of thiophene: A review. Der Pharma Chemica 2011, 3, (4), 38-54.

[S12] Elkins, G., THE EFFECT OF ETHYLENE GLYCOL ANTI-FREEZE ADDITIVE ON THE PERFORMANCE OF WATER EXTINGUISHERS. Fire Safety Science 1964, 560, -1--1.

[S13] Li, R.; Miao, C.; Wang, Y., Purity analysis of industrial triethylene glycol by capillary gas chromatography. Journal of Chromatographic Science 1998, 36, (7), 349-351.

[S14] Yu, Y.-X.; He, M.-Y.; Gao, G.-H.; Li, Z.-C., Boiling points for five binary systems of sulfolane with aromatic hydrocarbons at $101.33 \mathrm{kPa}$. Fluid Phase Equilibria 2001, 190, (1), 6171. 


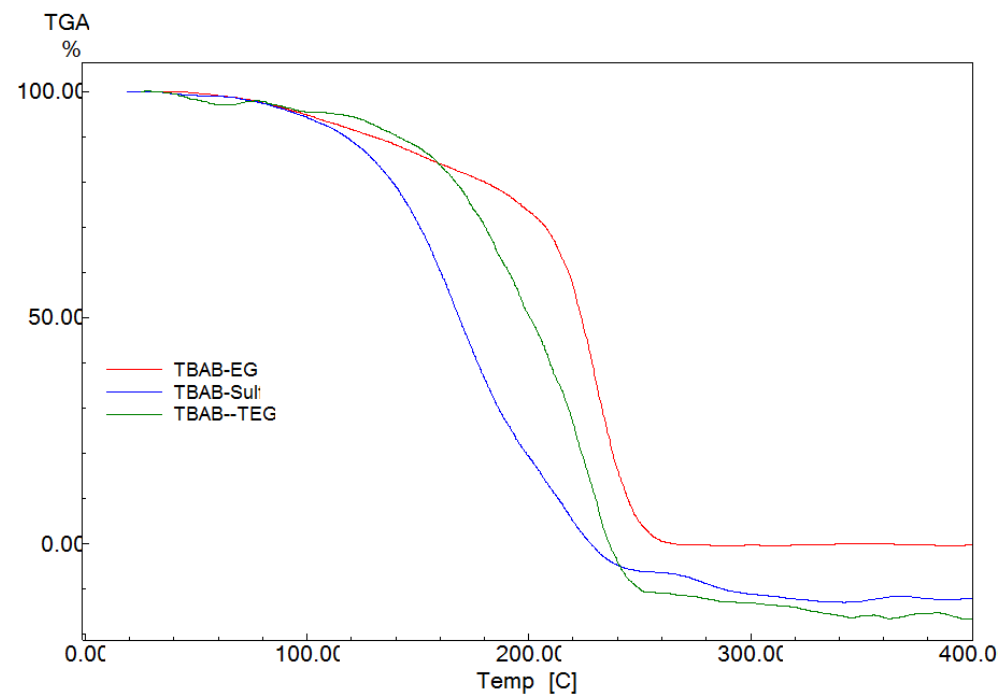

Fig. S1. TGA analysis of TBAB/EG (1:4), TBAB/TrEG (1:4) and TBAB/Sul (1:7) DESs

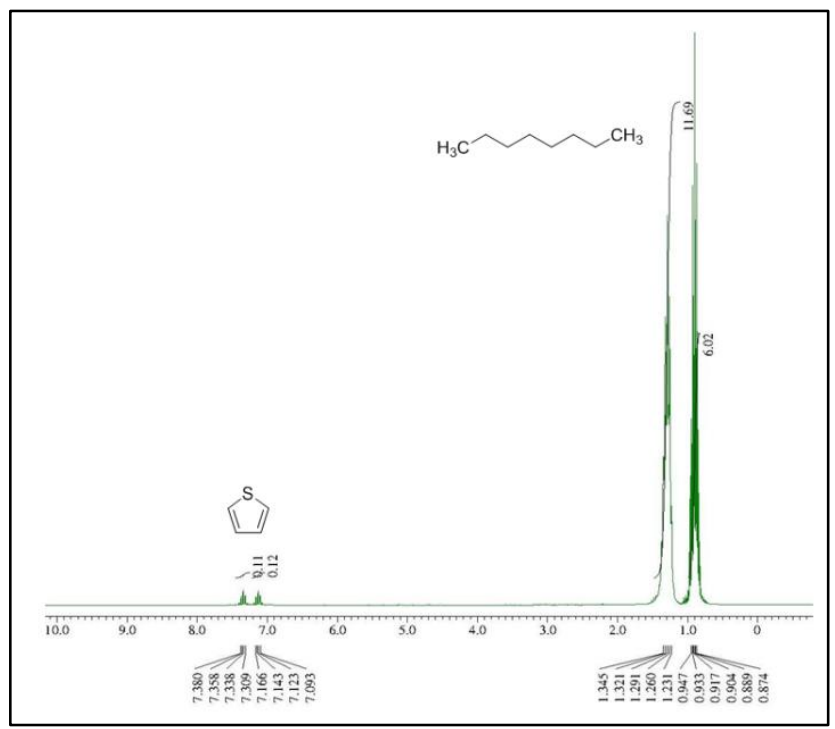

Fig. S2. ${ }^{1} \mathrm{H}$ NMR of the raffinate phase (no sulfolane is detected in the top layer) 


\section{Uncertainties estimation}

Regarding the statistical error/random error ${ }^{1}$, three samples were taken from each phase at equilibrium and analyzed using HPLC. The average value is the calculated and reported in the corresponding Table. An example for calculating the average and standard deviation for MeTPPBr/EG (1:4) DES is reported table S6.

Table S6. Standard deviation on measured solubilities with MeTPPBr/EG (1:4) DES

\begin{tabular}{|c|c|c|c|c|c|c|c|c|}
\hline \multicolumn{9}{|c|}{ Top Layer } \\
\hline \multicolumn{3}{|c|}{ Mol Fraction } & \multicolumn{3}{|c|}{ Average Mol Fraction } & \multicolumn{3}{|c|}{ STDEV Mol Fraction } \\
\hline Thiophene & nHeptane & $\begin{array}{c}\text { DES } \\
\mathbf{3} \\
\end{array}$ & Thiophene & Heptane & DES & Thiophene & Heptane & DES \\
\hline 0.105 & 0.895 & 0.000 & \multirow{3}{*}{0.105} & \multirow{3}{*}{0.895} & \multirow{3}{*}{0.000} & \multirow{3}{*}{0.003} & \multirow{3}{*}{0.003} & \multirow{3}{*}{0.000} \\
\hline 0.109 & 0.891 & 0.000 & & & & & & \\
\hline 0.102 & 0.898 & 0.000 & & & & & & \\
\hline 0.197 & 0.803 & 0.000 & \multirow{3}{*}{0.199} & \multirow{3}{*}{0.801} & \multirow{3}{*}{0.000} & \multirow{3}{*}{0.001} & \multirow{3}{*}{0.001} & \multirow{3}{*}{0.000} \\
\hline 0.200 & 0.800 & 0.000 & & & & & & \\
\hline 0.200 & 0.800 & 0.000 & & & & & & \\
\hline 0.288 & 0.712 & 0.000 & \multirow{3}{*}{0.288} & \multirow{3}{*}{0.712} & \multirow{3}{*}{0.000} & \multirow{3}{*}{0.008} & \multirow{3}{*}{0.008} & \multirow{3}{*}{0.000} \\
\hline 0.299 & 0.701 & 0.000 & & & & & & \\
\hline 0.278 & 0.722 & 0.000 & & & & & & \\
\hline 0.393 & 0.607 & 0.000 & \multirow{3}{*}{0.393} & \multirow{3}{*}{0.607} & \multirow{3}{*}{0.000} & \multirow{3}{*}{0.001} & \multirow{3}{*}{0.001} & \multirow{3}{*}{0.000} \\
\hline 0.392 & 0.608 & 0.000 & & & & & & \\
\hline 0.393 & 0.607 & 0.000 & & & & & & \\
\hline 0.490 & 0.510 & 0.000 & \multirow{3}{*}{0.490} & \multirow{3}{*}{0.510} & \multirow{3}{*}{0.000} & & & \\
\hline 0.489 & 0.511 & 0.000 & & & & 0.001 & 0.001 & 0.000 \\
\hline 0.491 & 0.509 & 0.000 & & & & & & \\
\hline 0.583 & 0.417 & 0.000 & & & & & & \\
\hline 0.581 & 0.419 & 0.000 & 0.583 & 0.417 & 0.000 & 0.001 & 0.001 & 0.000 \\
\hline 0.583 & 0.417 & 0.000 & & & & & & \\
\hline 0.686 & 0.314 & 0.000 & & & & & & \\
\hline 0.683 & 0.317 & 0.000 & 0.685 & 0.315 & 0.000 & 0.001 & 0.001 & 0.000 \\
\hline 0.685 & 0.315 & 0.000 & & & & & & \\
\hline 0.797 & 0.203 & 0.000 & & & & & & \\
\hline 0.796 & 0.204 & 0.000 & 0.797 & 0.203 & 0.000 & 0.001 & 0.001 & 0.000 \\
\hline 0.798 & 0.202 & 0.000 & & & & & & \\
\hline
\end{tabular}

1 These errors are caused by unpredictable fluctuations in the environmental conditions, instruments functioning and experimenter behavior. The related uncertainty is evaluated by statistical means by repeating the measurement. These uncertainties are of type $\mathbf{A}$ and are represented by the standard deviation $\boldsymbol{u}_{\boldsymbol{A}}$. 


\begin{tabular}{|c|c|c|c|c|c|c|c|c|}
\hline \multicolumn{9}{|c|}{ Bottom Layer } \\
\hline \multicolumn{3}{|c|}{ Mol Fraction } & \multicolumn{3}{|c|}{ Average Mol Fraction } & \multicolumn{3}{|c|}{ STDEV Mol Fraction } \\
\hline Thiophene & nHeptane & $\begin{array}{c}\text { DES } \\
\mathbf{3}\end{array}$ & Thiophene & Heptane & DES & Thiophene & Heptane & DES \\
\hline 0.038 & 0.017 & 0.945 & \multirow{3}{*}{0.039} & \multirow{3}{*}{0.017} & \multirow{3}{*}{0.944} & \multirow{3}{*}{0.001} & \multirow{3}{*}{0.001} & \multirow{3}{*}{0.001} \\
\hline 0.041 & 0.017 & 0.942 & & & & & & \\
\hline 0.039 & 0.016 & 0.945 & & & & & & \\
\hline 0.071 & 0.008 & 0.921 & \multirow{3}{*}{0.071} & \multirow{3}{*}{0.008} & \multirow{3}{*}{0.921} & \multirow{3}{*}{0.001} & \multirow{3}{*}{0.001} & \multirow{3}{*}{0.001} \\
\hline 0.072 & 0.007 & 0.921 & & & & & & \\
\hline 0.070 & 0.008 & 0.922 & & & & & & \\
\hline 0.084 & 0.008 & 0.908 & \multirow{3}{*}{0.084} & \multirow{3}{*}{0.008} & \multirow{3}{*}{0.908} & \multirow{3}{*}{0.003} & \multirow{3}{*}{0.001} & \multirow{3}{*}{0.002} \\
\hline 0.080 & 0.009 & 0.911 & & & & & & \\
\hline 0.087 & 0.007 & 0.906 & & & & & & \\
\hline 0.106 & 0.010 & 0.884 & \multirow{3}{*}{0.106} & \multirow{3}{*}{0.010} & \multirow{3}{*}{0.884} & \multirow{3}{*}{0.002} & \multirow{3}{*}{0.001} & \multirow{3}{*}{0.003} \\
\hline 0.109 & 0.011 & 0.880 & & & & & & \\
\hline 0.104 & 0.009 & 0.887 & & & & & & \\
\hline 0.138 & 0.007 & 0.855 & \multirow{3}{*}{0.138} & \multirow{3}{*}{0.007} & \multirow{3}{*}{0.855} & & & \\
\hline 0.140 & 0.009 & 0.852 & & & & 0.002 & 0.001 & 0.003 \\
\hline 0.136 & 0.006 & 0.858 & & & & & & \\
\hline 0.157 & 0.005 & 0.838 & & & & & & \\
\hline 0.159 & 0.006 & 0.834 & 0.157 & 0.005 & 0.838 & 0.002 & 0.001 & 0.003 \\
\hline 0.155 & 0.004 & 0.841 & & & & & & \\
\hline 0.172 & 0.004 & 0.824 & & & & & & \\
\hline 0.173 & 0.005 & 0.822 & 0.172 & 0.004 & 0.824 & 0.001 & 0.001 & 0.002 \\
\hline 0.171 & 0.004 & 0.826 & & & & & & \\
\hline 0.202 & 0.005 & 0.793 & & & & & & \\
\hline 0.204 & 0.006 & 0.791 & 0.202 & 0.005 & 0.793 & 0.001 & 0.001 & 0.002 \\
\hline 0.201 & 0.004 & 0.795 & & & & & & \\
\hline
\end{tabular}

As can be seen in this table, the maximum error is estimated to be \pm 0.008 in mole fraction, while the average uncertainty doesn't exceed \pm 0.002 .

Then, in addition to this statistical error, we can consider the inherent uncertainty ${ }^{2}$ due to the calibration of the HPLC instrument. The calibration curve of thiophene (given below in figure 1 and added to the Supporting Information) shows and excellent linearity (with $R^{2}=0.96603$ ) through the whole range of concentration.

\footnotetext{
${ }^{2}$ These errors usually come from the measuring instrument or from the method. Some examples: Offset or zero setting error, manufacturer precision, scale factor error, calibration ... etc. Systematic errors are linked to the instrument accuracy. These uncertainties are of type $\mathbf{B}$.
} 


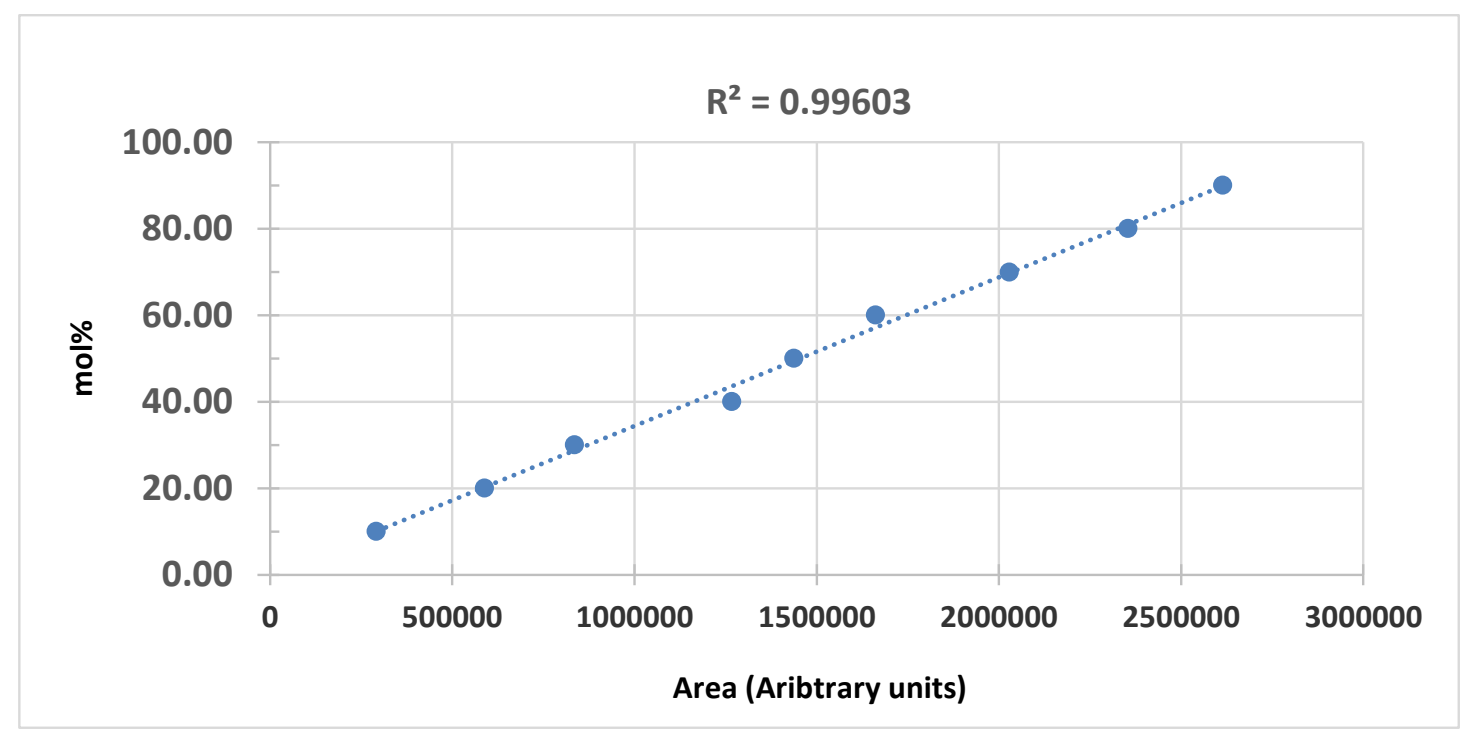

Figure S3. HPLC calibration curve of thiophene

This regression curve provides us with the standard deviation for the regression parameter $a\left(\sigma_{A}\right)$. Our calculation revealed that the standard deviation of this calibration is 0.0092 .

\section{$\underline{\text { Error propagation }}$}

In this section we describe how the propagation of errors from the individual uncertainties to the calculated value is calculated.

Indeed, if a variable $\mathrm{Z}$ is a function of other directly measured variable $\mathrm{X}$ and $\mathrm{Y}, Z=f(X, Y)$

Then, the uncertainty is calculated as follow

$$
u_{Z}=\sqrt{\left(\frac{\partial f}{\partial X} \cdot u_{X}\right)^{2}+\left(\frac{\partial f}{\partial Y} \cdot u_{Y}\right)^{2}}
$$

Let us consider the example of the distribution ratio $D$. The value of $D_{\text {thio }}$ is defined as the ratio of thiophene concentration in the extract phase to that in the raffinate phase, as shown in Equation (2), where $x_{\text {thio }}$ is the thiophene concentration in mole fraction, and the superscripts bottom and top refers to the extract and raffinate phases, respectively.

$$
D_{\text {thio }}=x_{\text {thio }}^{\text {bottom }} / x_{\text {thio }}^{\text {top }}
$$

Then by applying equation (1), the uncertainty on distributed ratio is calculated by the following expression 


$$
U=\frac{x_{\text {thio }}^{\text {bottom }}}{x_{\text {thio }}^{\text {top }}} \sqrt{\frac{U_{x}{ }^{2}}{\left(x_{\text {thio }}^{\text {bottom }}\right)^{2}}+\frac{U_{x}{ }^{2}}{\left(x_{\text {thio }}^{\text {top }}\right)^{2}}}
$$

Table S7. Propagation error on distribution ratio

\begin{tabular}{|c|c|c|c|c|c|c|c|c|}
\hline \multicolumn{3}{|c|}{ Top layer } & \multicolumn{3}{|c|}{ Bottom layer } & \multirow{2}{*}{$D_{\text {thio }}$} & \multirow{2}{*}{$\begin{array}{c}\boldsymbol{U}_{\boldsymbol{x}}=\mathbf{0 . 0 0 8} \\
\boldsymbol{U}_{\boldsymbol{D}}\end{array}$} & \multirow{2}{*}{$\begin{array}{c}\boldsymbol{U}_{\boldsymbol{x}}=\mathbf{0 . 0 0 2} \\
\boldsymbol{U}_{\boldsymbol{D}}\end{array}$} \\
\hline Thiophene & heptane & DES3 & Thiophene & heptane & DES3 & & & \\
\hline 0.105 & 0.895 & 0.000 & 0.039 & 0.017 & 0.944 & 0.373 & 0.081 & 0.020 \\
\hline 0.199 & 0.801 & 0.000 & 0.071 & 0.008 & 0.921 & 0.356 & 0.043 & 0.011 \\
\hline 0.288 & 0.712 & 0.000 & 0.084 & 0.008 & 0.908 & 0.290 & 0.029 & 0.007 \\
\hline 0.393 & 0.607 & 0.000 & 0.106 & 0.010 & 0.884 & 0.271 & 0.021 & 0.005 \\
\hline 0.490 & 0.510 & 0.000 & 0.138 & 0.007 & 0.855 & 0.282 & 0.017 & 0.004 \\
\hline 0.583 & 0.417 & 0.000 & 0.157 & 0.005 & 0.838 & 0.270 & 0.014 & 0.004 \\
\hline 0.685 & 0.315 & 0.000 & 0.172 & 0.004 & 0.824 & 0.251 & 0.012 & 0.003 \\
\hline 0.797 & 0.203 & 0.000 & 0.202 & 0.005 & 0.793 & 0.254 & 0.010 & 0.003 \\
\hline
\end{tabular}

In this table we have performed the calculations with considering the maximum error $\left(U_{x}=0.008\right)$ and the average error $\left(U_{x}=0.002\right)$ and we can clearly see the impact on the distribution ratio uncertainty. 